\title{
Treating anxiety complicated by substance misuse
}

\author{
Anne Lingford-Hughes, John Potokar \\ \& David Nutt
}

The relationship between anxiety disorders and substance misuse is intimate. Here we concentrate on alcohol, although for completeness we also briefly mention other substances taken for anxiety relief or that cause anxiety.

The term 'misuse' is important and should be qualified. Many people drink alcohol or take illicit drugs without any apparent consequences. Once this use becomes problematic, at any level of consumption, misuse is a more appropriate term.

Recent research exploring the molecular underpinning of anxiety disorders, together with advances in our understanding of how alcohol affects brain neurochemistry, can help to explain this intimate relationship. We review the evidence and how this can provide a framework for both diagnosis and management in a patient with comorbidity. Few studies address the best way of managing patients with dual diagnosis but several drugs are licensed for the treatment of individual anxiety disorders and are likely to be helpful in combination with alcohol treatment strategies.

Until recently, anxiety was used as an umbrella term that spanned the spectrum from normal human emotion to severe pathological states. The 1970 s and 1980s heralded the beginning of a more rigorous approach to the discrete constellation of symptoms that make up the individual anxiety disorders. This has not only helped in tailoring therapeutic approaches but has also enabled researchers to explore the pathophysiological foundations of the various disorders. The ICD-10 (World Health
Organization, 1992) categorises anxiety disorders as phobic (social, simple, agoraphobic), generalised, panic disorder and obsessive-compulsive disorder (OCD). Of these, the last is less robustly associated with substance misuse.

Although pharmacological and psychological treatments for anxiety disorders are effective, the most common response for an individual with anxiety is to use recreational drugs, principally alcohol, to alleviate the symptoms. The widespread availability and relative low cost of alcohol means that although it is widely used as a social lubricant and for its short-term euphoriant effects, it is also frequently used as self-medication for anxiety disorders, low mood and sleep problems. Prospective studies show that alcohol dependence and anxiety disorders demonstrate a reciprocal causal relationship over time, with anxiety disorders leading to alcohol dependence and vice versa (Kushner et al, 1990).

\section{Psychoactive substances and anxiety}

The ICD-10 lists a number of behavioural and mental disorders (e.g. acute intoxication, dependence and withdrawal) that can result from misuse of psychoactive substances, including alcohol and other sedatives, opioids, cannaboids, stimulants such as cocaine, amphetamine and caffeine,

Anne Lingford-Hughes and John Potokar are senior lecturers at the University of Bristol. Dr Lingford-Hughes is Honorary Consultant Psychiatrist at the Bristol Area Specialist Alcohol Service (Avon and Wiltshire Partnership Mental Health Trust) and Dr Potokar is Honorary Consultant in Liaison Psychiatry at the Bristol Royal Infirmary. Professor David Nutt is currently Professor of Psychopharmacology, Head of the Department of Clinical Medicine and Dean of Clinical Medicine and Dentistry, based at the University of Bristol (Psychopharmacology Unit, School of Medical Sciences, University of Bristol, Bristol BS8 1TD, UK; tel: 0117925 3066; fax: 0117927 7057; e-mail: David.J.Nutt@bristol.ac.uk). The Psychopharmacology Unit's research programme includes anxiety disorders, addiction and neuroimaging. 
Table 1 Anxiety as a feature of substance misuse

\begin{tabular}{|c|c|c|}
\hline Substance & Use & Intoxication \\
\hline Alcohol & $\begin{array}{l}\text { To overcome anxiety } \\
\text { (especially social) }\end{array}$ & \\
\hline $\begin{array}{l}\text { Stimulants } \\
\text { (cocaine, } \\
\text { amphetamine) }\end{array}$ & $\begin{array}{l}\text { To overcome social } \\
\text { anxiety }\end{array}$ & $\begin{array}{l}\text { Anxiety with } \\
\text { tachycardia, pupillary } \\
\text { dilation, psychomotor } \\
\text { agitation, impaired } \\
\text { judgement, impulsive } \\
\text { behaviour }\end{array}$ \\
\hline Hallucinogens & & $\begin{array}{l}\text { Anxiety, with altered } \\
\text { perceptions, } \\
\text { hallucinations }\end{array}$ \\
\hline PCP & & $\begin{array}{l}\text { Occasionally anxiety, } \\
\text { with usually delirious } \\
\text { psychotic state }\end{array}$ \\
\hline
\end{tabular}

$\begin{array}{ll}\text { Withdrawal } & \text { Long-term effects } \\ \begin{array}{l}\text { Pronounced anxiety } \\ \text { (see text) }\end{array} & \begin{array}{l}\text { Panic disorder and GAD } \\ \text { can emerge from misuse }\end{array} \\ \text { Yes } & \text { Panic disorder, phobias and } \\ & \text { GAD can emerge from misuse }\end{array}$

Inhalants

GAD and panic disorder can emerge from chronic use

Nicotine

Anxiety

Pronounced anxiety

Caffeine

Rarely used for anxiety relief

Not clear, but flashbacks can be similar to symptoms of PTSD

Opioids

Cannabis

$$
\begin{aligned}
& \text { Can present like a } \\
& \text { panic attack but with } \\
& \text { paranoid thoughts; } \\
& \text { more likely in } \\
& \text { inexperienced smokers }
\end{aligned}
$$

GAD, generalised anxiety disorder; PTSD, post-traumatic stress disorder; PCP, 1-(1-phencyclohexyl)piperidine, phencyclidine.

hallucinogens and tobacco. The interaction between anxiety and other substance misuse is described in Table 1.

\section{Alcohol and other sedatives}

Although alcohol is a fast-acting and effective anxiolytic, it can also lead to increased anxiety, particularly when consumption is excessive. Withdrawal is experienced as anxiety, thus fuelling more alcohol intake, which results in a vicious cycle of anxiety and alcohol consumption (Fig. 1). Patients with panic disorder who are alcohol dependent are unable to distinguish panic symptoms, with the exception of tremor, from alcohol withdrawal (George et al, 1988).

Anxiety commonly presents as a symptom of alcohol withdrawal, initially in the form of 'shakes and sweats' as the blood alcohol level declines. Raistrick (2000) has recently reviewed the management of alcohol withdrawal.
Alcohol shares many similar pharmacological and behavioural actions with benzodiazepines and barbiturates. These drugs can be prescribed as sedatives, anxiolytics, hypnotics or anticonvulsants. Short-acting benzodiazepines have greater addictive potential than longer-acting formulations. Not surprisingly, anxiety is commonly associated with withdrawal from benzodiazepines. At what dose

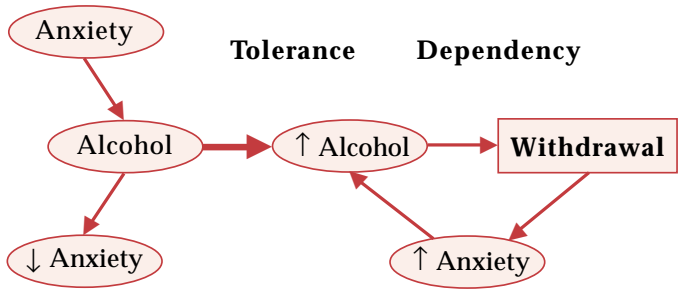

Fig. 1 Pathway from anxiety to self-medication with alcohol and consequent dependency;

$\uparrow$, increase; $\downarrow$, decrease 
and after how long a benzodiazepine causes significant withdrawal is difficult to define in routine clinical practice but current British National Formulary (BNF; British Medical Association \& Royal Pharmaceutical Society of Great Britain, 2001) guidelines suggest prescription only for 'disabling' conditions and for not longer than 4 weeks. However, it is important to note that benzodiazepines are far safer than alcohol and in patients with anxiety disorders long-term use is sometimes indicated where other medications have failed.

\section{Alcohol misuse and anxiety: prevalence and incidence}

Alcoholism and social anxiety disorder comprise the next most prevalent mental disorders after depression (Kessler et al, 1997). They are often present together, increasing the therapeutic challenge.

There have been several community prevalence studies determining the comorbidity of anxiety and alcoholism. People with an anxiety disorder have approximately double the odds of also having a substance misuse disorder (Table 2). This typically equates to one-quarter to one-third of people with an anxiety disorder, with rates of comorbidity being higher in women. The anxiety disorder generally precedes alcohol or drug problems or dependence. This finding is consistent but not confirmatory of the 'self-medication hypothesis'. The exception to this is panic disorder, which can emerge from alcohol misuse (Merikangas et al, 1998).

In patient populations, the lifetime prevalence of alcohol misuse or dependence in people with social anxiety disorder is $22 \%$, with the prevalence of social anxiety disorder in people seeking treatment for alcohol problems around 15\% (Regier et al, 1990). The rates of any anxiety disorders in in-patients with alcohol dependence ranges from 22.6 to $68.7 \%$, with phobias typically highly represented (Kushner et al,
1990). Therefore about one-quarter to one-fifth of patients diagnosed with alcohol problems or social anxiety disorder will also suffer from the other disorder. In people with DSM-III-R alcohol abuse or dependence, the prevalence of social anxiety disorder in females is higher than in males (female:male ratio $=30 \%: 19 \%$; Kessler et al, 1994, 1997).

The rates of anxiety disorders in substance misusers are also quite high. In a survey of drug misusers in contact with treatment services, the lifetime prevalence of a phobic disorder was $26 \%$ in men and $45 \%$ in women (Krausz et al, 1998). For other anxiety disorders the rates were $10 \%$ for men and $22 \%$ for women. In an out-patient methadonemaintained population, lifetime rates of any anxiety disorder were $6.1 \%$ in men and $10.7 \%$ in women. The most common disorders seen were simple phobia (females) and panic disorder (males) (Brooner et al, 1997).

Thus, in both substance misuse and anxiety disorder populations, rates of the other disorders are high. When comorbidity occurs it is likely that each disorder will be more difficult to treat and consequently the clinician needs to know not only why this relationship occurs but also how to recognise and manage this comorbidity.

\section{Aetiological theories and issues}

The co-occurrence of alcohol and anxiety has led to proposals of an aetiological link. Both biological and psychosocial factors have been described. The concept of alcohol as a provider of 'Dutch courage' to help people with social anxiety attend social occasions is widely held. This self-medication hypothesis receives support from prevalence surveys (Merikangas et al, 1998) showing that phobias and social anxiety tend to have preceded the onset of alcohol or drug misuse. On the other hand, panic disorder does not necessarily follow this pattern and in some patients it clearly emerges in alcohol dependence.

Table 2 Odds ratio of an additional diagnosis (alcohol or drug problems or dependence) with any anxiety disorder

$\begin{array}{lcccc}\text { Survey } & \text { Alcohol problems } & \text { Alcohol dependence } & \text { Drug problems } & \text { Drug dependence } \\ \text { ECA } & 1 & 1.8 & 2.3 & 2.4 \\ \text { NCS } & & & & \\ \text { Male } & 0.95 & 2.2 & & \\ \text { Female } & 1.78 & 3.08 & 2.95 & 4.78\end{array}$

ECA, Epidemiologic Catchment Area (Regier et al, 1990); NCS, National Comorbidity Survey (Kessler et al, 1997); ICPE, International Consortium in Psychiatric Epidemiology (Merikangas et al, 1998). 
The association does not necessarily reflect a direct causal relationship. Instead, the vulnerability to both disorders may arise from another factor, perhaps genetic, familial or biological.

A number of research paradigms have been devised to study the anxiolytic effects of alcohol. Not all studies confirm that alcohol is anxiolytic, and factors such as how anxiety is defined (e.g. as tension, fear, frustration, stress), the amount of alcohol consumed, previous experience, situation and expectations play a role (Kushner et al, 1990). For example, in patients with social anxiety disorder, alcohol itself failed to reduce anxiety associated with public speaking but the belief that they had received alcohol did reduce anxiety (Himle et al, 1999).

From a psychiatric perspective the relationship between alcohol and anxiety disorders cannot be understood without knowledge of the effect of alcohol on brain neurotransmission and the role of neurotransmitters in these disorders. In the past it was thought that anxiety disorders were purely functional, with no real biological basis, and alcohol dependence was often perceived to be the result of constitutional weakness. It is now very clear that neurotransmitter and receptor function are altered in both anxiety disorders and alcohol dependence. Three neurotransmitter systems are likely to be especially important in this relationship: the $\gamma$ aminobutyric acid (GABA), serotonin (5-HT) and noradrenaline systems.

\section{The GABA-ergic system}

Although alcohol acts on a variety of neurotransmitter systems (see Nutt, 1999), its effects on the GABAbenzodiazepine receptor (GBzR), are likely to be critical in its anxiolytic effect. This receptor also appears important in the tolerance, dependence and withdrawal that can occur through use of alcohol or benzodiazepines. GABA is the major inhibitory neurotransmitter system in the central nervous system and the GBzR has several different functional forms, which differ in their sensitivity to alcohol or benzodiazepines. For instance, by altering the $\alpha 1$ subunit of the GBzR in mice, diazepam is no longer sedative and by altering the $\alpha 2$ subunit, it is no longer anxiolytic (Rudolph et al, 2001).

As alcohol is a GBzR agonist, it has been proposed that hypofunction of this system is involved in vulnerability to alcoholism. Although short-term exposure to alcohol increases GABA-ergic function, long-term exposure is associated with reduced GBzR levels and function. It has been proposed that such a reduction is important as an underlying neurobiological mechanism of tolerance. Neuroimaging studies have shown reduced levels of the GBzR in abstinent alcohol-dependent patients, predominantly in the frontal lobes (e.g. Lingford-Hughes et al, 1998).

There is also evidence for altered GABA-ergic function in anxiety disorders. Patients with panic disorder appear to be sub-sensitive to the effects of benzodiazepines (Roy-Byrne et al, 1989), suggesting an alteration in the 'set-point' of the receptor. This, together with the panicogenic effects of the benzodiazepine antagonist flumazenil, points to altered GABA-ergic function, which may underpin the raised anxiety in these patients. Chronic benzodiazepine use in patients with anxiety disorders is associated with reduced GBzR sensitivity (Potokar et al, 1999) and we have some preliminary evidence that alcohol dependency is associated with grossly reduced GBzR sensitivity. Furthermore, Malizia et al (1998) have shown, using positron emission tomography, that there is a reduction in global GBzR binding in patients with panic disorder compared with matched controls, emphasising the important role of this receptor system in this disorder.

\section{The serotonergic system}

Several lines of evidence point to an important role of 5-HT in anxiety and alcoholism (Nutt \& Cowen, 1987; Heinz et al, 2001). Clinically, a number of serotonergic drugs are effective in treating anxiety disorders and many are licensed (see below and Table 3).

In alcoholism, low 5-HT function is associated with Type 2 alcoholism, in which early disease onset, impulsivity, antisocial personality traits and a positive family history are seen (Heinz et al, 2001). In this alcohol-dependent population, increased 5HT (with m-chlorophenylpiperazine (mCPP), a mixed 5-HT agonist/antagonist) resulted in craving and an increased urge to drink alcohol (George et al, 1997) (Fig. 2).

\section{The noradrenergic system}

Enhanced noradrenergic activity is thought to underlie many symptoms of drug or alcohol withdrawal, including agitation, anxiety, sweating and tachycardia (George et al, 1990). These symptoms can be controlled with lofexidine, an $\alpha 2$ agonist, which reduces noradrenergic output from the brainstem. This drug is increasingly used to manage opiate withdrawal, but a recent study has suggested 
lack of efficacy in patients undergoing in-patient alcohol detoxification (Keaney et al, 2001).

A dysfunctional noradrenergic system is also implicated in anxiety, providing another common biological substrate for both anxiety and substance misuse. Drugs such as amphetamines and cocaine, which increase noradrenaline, are anxiogenic, while the $\alpha 2$ agonist clonidine reduces anxiety and has some activity in reducing spontaneous and lactateinduced panic (Coplan et al, 1992).

In conclusion it is clear that anxiety and alcohol misuse can each give rise to the other, but the relationship is complex. Similarities in the underlying cause of substance misuse and anxiety disorders suggest that a common mechanism may be effective in ameliorating both disorders.

\section{Management}

Three factors are likely to be important in the symptomatic and functional improvement of the patient with comorbidity: whether anxiety disorder is diagnosed, the degree of alcohol misuse (e.g. hazardous, harmful or dependent) and whether the relationship between the two has been explored.

There are many reasons why people with anxiety disorders initiate and maintain alcohol misuse and why current management is not optimum. These include the availability of alcohol, failure of patients and doctors to recognise pathological anxiety and uncertainty or lack of knowledge about treatment options. Education of both patients and doctors is obviously critical if some of these factors are to be addressed.

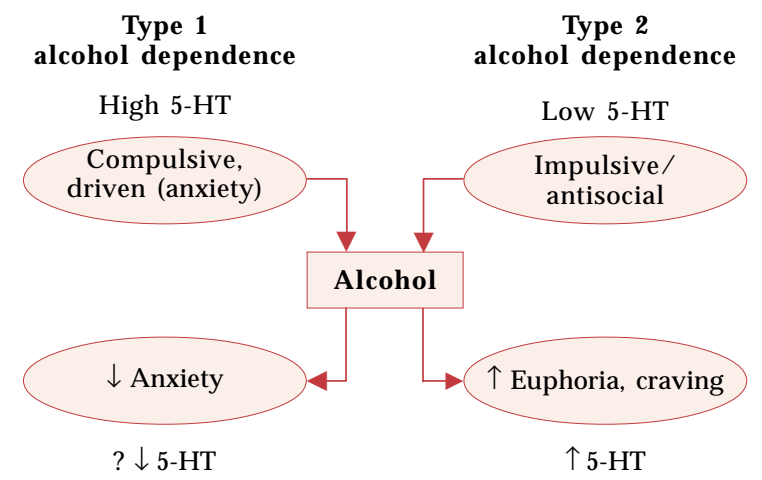

Fig. 2 Possible routes to alcohol dependence implicating the serotonergic (5-HT) system;

increase; $\downarrow$, decrease

\section{Diagnostic issues}

\section{Assessment of anxiety}

It is essential to establish whether a discrete anxiety disorder is present in patients with possible comorbidity. Three questions should be asked when assessing a patient 'with anxiety': is the patient suffering from an anxiety disorder; what type of anxiety disorder is it; and is there any comorbidity?

Anxiety is a normal human emotion that rises and falls in response to both external and internal cues. Pathological anxiety ('anxiety disorder') is characterised by its intensity, duration, autonomy (i.e. it has a life of its own) and functional impairment (often characterised by changes in behaviour).

If an anxiety disorder is suspected, are panic attacks present? Panic attacks are often not identified because the clinician asks only about the recent past, whereas the natural history of panic attacks frequently results in a reduction in their intensity with time. A useful question is to ask whether the patient has ever experienced a sudden surge in anxiety that felt overwhelming and was accompanied by the typical somatic and cognitive symptoms. If a positive answer is elicited, the clinician should ask about the first time that this ever happened - this will often be remembered in great detail and may also explain consequent avoidant (agoraphobic) behaviour. If a history of panic attacks is obtained, are these cued (social anxiety disorder, post-traumatic stress disorder (PTSD) or specific phobia) or spontaneous (panic disorder)?

Every patient who presents with anxiety symptoms should be asked about alcohol intake and, if a history of excessive alcohol use is obtained (hazardous, harmful or dependent), anxiety disorders should be screened for. A useful tool is the Alcohol Use Disorders Identification Test (AUDIT) questionnaire, which has been recommended for routine use by the World Health Organization (Saunders et al, 1993).

It is essential to try to establish the sequence of development of clinical problems. Was the anxiety disorder present before the alcohol misuse (e.g. social anxiety disorder, agoraphobia) or secondary to it (e.g. panic disorder, general anxiety disorder)? Psychoeducation is useful. Often patients will never have discussed in detail their anxiety symptoms and may not relate their alcohol problem to anxiety.

\section{Assessment of substance misuse}

Clearly, a comprehensive assessment of any patient is critical. Although it is commonly thought that patients do not accurately reveal their true alcohol 
and drug consumption, this is not necessarily the case nor should it hinder a history being obtained. Evidence can also be obtained from a physical examination: needle or track marks may be indicative of intravenous drug misuse and palmar erythema of alcohol misuse. A urine drug screen will reveal recent drug use; abnormal liver function tests and mean corpuscular volume are generally apparent in alcohol misuse. In patients with anxiety disorder, the amount being consumed is not as relevant as the reliance on the substance.

\section{Treatment}

\section{Anxiety disorders}

The following is a brief summary of the principal treatments for uncomplicated anxiety disorders. Table 3 summarises pharmacological treatments and potential problems with them. For a recent review see Argyropoulos et al (2000).

\section{Panic disorder}

Pharmacological treatments Results from several studies show that $70-80 \%$ of patients treated with anti-panic agents show moderate to marked improvement (e.g. Ballenger et al, 1993). Selective serotonin reuptake inhibitors (SSRIs) are efficacious; citalopram and paroxetine are licensed. Of the tricyclic antidepressants, clomipramine and imipramine are the most studied and are effective. There is slightly more evidence for clomipramine, which is the more serotonergic of these drugs. Improvement does not depend on affective status. None of the tricyclics are licensed for this indication in the UK. Most studies of monoamine oxidase inhibitors (MAOIs) have been uncontrolled, and although many clinicians view them as effective, they are usually reserved for treatment-resistant patients. Reversible monoamine oxidase inhibitors (RIMAs) such as moclobemide have minimal sideeffects and probably some efficacy. They are not licensed for use in panic disorder.

Benzodiazepines are efficacious but high doses of standard benzodiazepines or high-potency agents

\section{Table 3 Indications for pharmacotherapy and potential problems of prescribed drugs ${ }^{1}$}

\begin{tabular}{|c|c|c|}
\hline Drug & Anxiety disorder: indications & Potential problems \\
\hline $\begin{array}{l}\text { Selective serotonin } \\
\text { reuptake inhibitors }\end{array}$ & $\begin{array}{l}\text { Panic disorder } \\
\text { Generalised anxiety disorder } \\
\text { Obsessive-compulsive disorder } \\
\text { Post-traumatic stress disorder } \\
\text { Social anxiety disorder }\end{array}$ & $\begin{array}{l}\text { Possibly less efficacious } \\
\text { in Type } 2 \text { alcoholism }\end{array}$ \\
\hline Nefazodone & No specific indication & \\
\hline Trazodone & & $\begin{array}{l}\text { Enhanced sedative effects with } \\
\text { alcohol }\end{array}$ \\
\hline \multirow[t]{2}{*}{ Tricyclic antidepressants } & Panic disorder & $\begin{array}{l}\text { Enhanced sedative effects with } \\
\text { alcohol }\end{array}$ \\
\hline & Post-traumatic stress disorder & $\begin{array}{l}\text { Toxicity in overdose - often taken } \\
\text { with alcohol } \\
\text { Lowering of seizure threshold }\end{array}$ \\
\hline Venlafaxine (SNRI) & Generalised anxiety disorder & \\
\hline Reboxetine (NaRI) & No specific indication & \\
\hline Mirtazapine (NaSSA) & No specific indication & $\begin{array}{l}\text { Enhanced sedative effects with } \\
\text { alcohol }\end{array}$ \\
\hline Monoamine oxidase inhibitors & Social anxiety disorder & Potential interactions with alcohol \\
\hline Benzodiazepines & $\begin{array}{l}\text { Anxiety disorders } \\
\text { (disabling, <4 weeks use) }\end{array}$ & $\begin{array}{l}\text { Dependence with escalating use of } \\
\text { alcohol/drugs } \\
\text { Enhanced effects of alcohol }\end{array}$ \\
\hline Buspirone & Generalised anxiety disorder & $\begin{array}{l}\text { Enhanced sedative effects with } \\
\text { alcohol }\end{array}$ \\
\hline
\end{tabular}

SNRI, serotonin and noradrenaline reuptake inhibitor; NaRI, noradrenaline reuptake inhibitor; NaSSA, noradrenergic and specific serotonergic antidepressant.

1. Liver (and renal) impairment will affect dosing regimen for most drugs, and severe impairment often results in the drug being contraindicated (see current British National Formulary). Since many patients with anxiety disorders are sensitive to side-effects, pharmacotherapy should be started at a low dose and slowly increased. 
such as clonazepam are needed (presumably because of reduced GBzR sensitivity in these patients). Tolerance to therapeutic effects does not tend to occur. Their fast onset of action makes them ideal for co-prescribing when other agents (e.g. SSRIs) are initiated, thus preventing the initial exacerbation of symptoms that occurs with these agents. Relapse rates are high when they are withdrawn.

Psychological treatments Behavioural (both exposure and relaxation), cognitive and cognitive-behavioural treatments are effective. Psychodynamic therapy has not been evaluated.

\section{Post-traumatic stress disorder}

Pharmacological treatments Up to $80 \%$ of patients with PTSD have comorbid conditions. Antidepressants have the most evidence supporting use, especially those with serotonergic activity. SSRIs are useful and both paroxetine (UK) and sertraline (US) are licensed. Nefazodone and trazodone, which block 5- $\mathrm{HT}_{2}$ receptors as well as 5-HT reuptake, may be useful especially if there is pronounced sleep disturbance. MAOIs may be useful for treatmentrefractory cases. Although there are no controlled studies of benzodiazepines, clonazepam (which has a long duration of action and some serotonergic effects) is sometimes usefully prescribed.

Psychological treatments Cognitive-behavioural strategies (usually employing exposure rather than systematic desensitisation) are effective in reducing symptoms. Several studies have shown eye movement desensitisation and reprocessing to be reasonably effective (Rothbaum, 1997; Carlson et al, 1998), although other studies report more modest effects (Pitman et al, 1996; Devilly \& Spence, 1999).

\section{Social anxiety disorder}

Pharmacological treatments The SSRIs are efficacious, tolerable and safe and are generally considered firstline treatment. Paroxetine is licensed for treatment of social anxiety disorder. MAOIs are also effective but their relatively poor tolerability and safety means that they are reserved for refractory cases. Early studies (Versiani et al, 1992) suggested considerable efficacy for RIMAs but more recent studies (Schneier et al, 1998) have been equivocal, although moclobemide is licensed.

Two controlled studies of benzodiazepines in social anxiety disorder have been carried out (Davidson et al, 1993). A response rate of $78 \%$ was found for clonazepam (v. 20\% for placebo). Although well-tolerated, concerns about dependence mean that they are usually reserved for resistant cases.

Beta-blockers are frequently prescribed, especially by general practitioners, but are generally not useful in generalised social anxiety disorder. They are, however, effective in specific performance anxiety.

Psychological treatments Both cognitive therapy and exposure therapy are effective and the combination probably has advantages over each therapy alone.

\section{Generalised anxiety disorder (GAD)}

Pharmacological treatments Both trazodone and imipramine have demonstrated efficacy in controlled trials. Of the newer agents, venlafaxine is effective and has recently been licensed for this use. Buspirone is effective and may be especially so when there are prominent psychic symptoms. Benzodiazepines are effective and have rapid onset of action. Although patients with uncomplicated GAD do not tend to run into problems with benzodiazepine use, concerns about dependence limit their prescription.

Psychological treatments Cognitive-behavioural therapies (CBTs) incorporating relaxation training, cognitive therapy and image rehearsal have some efficacy.

\section{Specific phobia}

Generally, drug treatments are not useful unless the fear is so great that exposure therapy (the treatment of choice) is not possible.

\section{Obsessive-compulsive disorder}

Pharmacological treatments Clomipramine is effective, as are the SSRIs. The relatively benign side-effect profile of the latter means that they are first-line treatments; paroxetine, fluoxetine, sertraline and fluvoxamine are licensed for this indication.

Psychological treatments Behaviour therapy is superior to both relaxation therapy and placebo. Cognitive therapy (especially CBT) appears to be effective.

\section{Treating anxiety disorders in the presence of substance misuse}

Treating comorbid substance misuse and anxiety can be tackled either by concentrating on one of the disorders first (but which one?), or by addressing them together. Psychological approaches are commonly applied to treat both substance misuse and many forms of anxiety, although lack of availability may be a limiting factor. Pharmacotherapy is frequently used to treat anxiety but some form of psychological therapy is also usually required. In treating substance misuse, pharmacotherapy can be used as a substitute, for withdrawal or for maintaining abstinence. 
Although both psychological and pharmacological treatments have been shown to be efficacious in treating either anxiety or a substance misuse disorder, there is limited knowledge about treating the comorbid condition (Scott et al, 1998). Most of the literature concerned with treating comorbid anxiety disorder concentrates on alcohol misuse, with little described on drug misuse. Clearly, if alcohol is being used to 'medicate' anxiety symptoms, then relapse after or during alcohol treatment is more likely if the anxiety disorder is not also treated. A pragmatic approach might be to target treatment on the patient's anxiety, even if he or she is still drinking, but in our experience this is more likely to have an impact if drinking is at non-dependent levels.

\section{Abstinence}

Abstinence alone may improve anxiety symptoms. Although $40 \%$ of in-patients undergoing alcohol withdrawal had significantly elevated anxiety ratings in the first week, in the following week scores returned to normal (Brown et al, 1991). This emphasises the need first to detoxify the patient and then to reassess after 2-3 weeks of sobriety (see UK Alcohol Forum guidelines at http:// www.ukalcoholforum.org). Even if abstinence is not achieved, minimising the contribution of alcohol or other drugs to the mental state is desirable.

\section{Psychological treatment}

Psychological strategies dominate treatment of alcohol and substance misuse, often involving group work. Inevitably, those with anxiety disorders, especially social anxiety disorder, find groups hard to attend. Thus, at best these patients may not be able to benefit fully from the treatment offered for alcoholism and at worst the underlying cause of their alcoholism may be untreated. Many alcohol services run anxiety-management groups or tackle a patient's anxiety in individual therapy.

Given that achieving abstinence can be very difficult for patients because it involves removing their 'anxiolytic', is there any evidence to support concurrent treatment? A recent study addressed this question: people with alcoholism were randomly assigned to 12 weeks of CBT for their alcohol dependence alone or for alcohol and social anxiety disorder together (Randall et al, 2001). Drinking behaviour and social anxiety improved in both groups, but the group that received treatment for both anxiety and alcohol had poorer outcomes in drinking behaviour. Notably, no correlation was seen between improvement in anxiety and drinking behaviour. The reasons for this result were not clear, but alcohol may have been drunk in order to cope with the treatment programme for anxiety. This study did not explicitly address whether treatment for alcohol misuse or for anxiety should come first. Another study compared out-patient CBT with 12step facilitation therapy in females with alcoholism and social anxiety disorder. Abstinence was achieved for longer with CBT than with 12-step therapy. However, the reverse was seen in males with the same disorder (Randall et al, 2000). These studies underscore the need for appropriate patient selection for treatment and that there is no universal panacea.

\section{Pharmacological treatment}

Almost 30 years ago imipramine was shown to improve panic disorder and drinking behaviour (Quitkin et al 1972), but few studies have been performed. Buspirone, a $5-\mathrm{HT}_{1 \mathrm{~A}}$ partial agonist, has also been shown to improve anxiety (some with GAD) in alcoholism and also to improve drinking behaviour and reduce craving (Kranzler et al, 1994). The impact of SSRIs in treating comorbid alcohol and anxiety is not clear.

When considering the use of SSRIs, it is important to recognise that subgroups of alcoholism do exist, in particular Types 1 and 2 (Cloninger et al, 1981). In treating alcoholism either with or without a comorbid depressive disorder, SSRIs in Type B (akin to Type 2) alcoholism may not only result in no improvement but may actually reduce the impact of psychological treatment such as CBT (Kranzler et $a l, 1996)$. Thus, it should be borne in mind that for some people, SSRIs are not the drug of choice.

The use of benzodiazepines in an alcoholdependent population is controversial (excluding use for alcohol withdrawal) and should not be undertaken without expert advice and monitoring. When abstinent, people with alcohol dependence may be at higher risk of benzodiazepine misuse and dependence because of their greater rewarding effects (Ciraulo \& Nace, 2000). Despite the level of concern, there is evidence to suggest that a history of alcohol misuse/dependence does not necessarily result in greater use of benzodiazepines, particularly for those who are less severely dependent. Therefore, their use should not be automatically excluded in the alcohol-misusing patient. Those patients who are severely dependent or with antisocial personality disorder or with polysubstance misuse are most at risk of misusing benzodiazepines. For some people, maintenance benzodiazepines can be indicated to maximise abstinence and minimise morbidity.

Although not specifically investigated in comorbid alcoholism and anxiety disorders, acamprosate may be useful. Most studies show that acamprosate as an adjunct to psychosocial treatment doubles the rate of abstinence, but clearly many patients are not 
helped. One study has suggested that patients who drink to overcome withdrawal or anxiety derived the greatest benefit (Lesch \& Walter, 1996).

\section{Conclusion}

Successful treatment of an anxiety disorder can result in improved drinking behaviour. However, many patients will continue to drink alcohol, thus impairing their chance of recovery. In the comorbid patient, drinking should be tackled first, but the anxiety disorder should be kept in mind and treatment for this should begin sooner rather than later. The picture is less clear for illicit drug misuse, with few data to guide management. Randomised controlled trials are needed to evaluate the efficacy of both psychological and pharmacological treatments, both singularly and together, in treating anxiety disorders complicated by substance misuse.

\section{References}

Argyropoulos, S. V., Sandford, J. J. \& Nutt, D. J. (2000) The psychobiology of anxiolytic drugs. Part 2: Pharmacological treatments of anxiety. Pharmacology and Therapeutics, 88, 213-227.

Ballenger, J. C. (1993) Panic disorder: efficacy of current treatments. Psychopharmacology Bulletin, 29, 477-486.

British Medical Association \& Royal Pharmaceutical Society of Great Britain (2001) British National Formulary (September issue). London \& Wallingford: BMJ Books and Pharmaceutical Press.

Brooner, R. K., King, V. L., Kidorf, M., et al (1997) Psychiatric and substance use comorbidity among treatment-seeking opioid abusers. Archives of General Psychiatry, 54, 71-80.

Brown, S. A., Irwin, M. \& Schuckit, M. A. (1991) Changes in anxiety among abstinent male alcoholics. Journal of Studies on Alcohol, 52, 55-61.

Carlson, J. G., Chemtob, C. M., Rusnak, K., et al (1998) Eye movement desensitization and reprocessing [EMDR] treatment for combat-related posttraumatic stress disorder. Journal of Traumatic Stress, 11, 3-24.

Ciraulo, A. D \& Nace, E. P. (2000) Benzodiazepine treatment of anxiety or insomnia in substance abuse patients. American Journal of Addiction, 9, 276-284.

Cloninger, C. R., Bohman, M. \& Sigvardsson, S. (1981) Inheritance of alcohol abuse. Cross-fostering analysis of adopted men. Archives of General Psychiatry, 38(8), 861868

Coplan, J. D., Liebowitz, M. R., Gorman, J. M., et al (1992) Noradrenergic function in panic disorder. Effects of intravenous clonidine pre-treatment on lactate induced panic. Biological Psychiatry, 31, 135-146.

Davidson, J. R., Potts, N., Richichi, E., et al (1993) Treatment of social phobia with clonazepam and placebo. Journal of Clinical Psychopharmacology, 13(6), 423-428.

Devilly, G. J. \& Spence, S. H. (1999) The relative efficacy and treatment distress of EMDR and a cognitive behavioural treatment protocol in the amelioration of post traumatic stress disorder. Journal of Anxiety Disorders,13, 131-157.

George, D. T., Zerby, A., Noble, S., et al (1988) Panic attacks and alcohol withdrawal: can subjects differentiate the symptoms? Biological Psychiatry, 24, 240-243.
-, Nutt, D. J., Dwyer, B. A., et al (1990) Alcoholism and panic disorder: is the comorbidity more than coincindence? Acta Psychiatrica Scandinavica, 81, 97-107.

- Benkelfat, C., Rawlings, R. R., et al (1997) Behavioural and neuroendocrine responses to $m$-chlorophenylpiperazine in subtypes of alcoholics and in healthy comparison subjects. American Journal of Psychiatry, 154, 81-87.

Heinz, A., Mann, K., Weinberger, D. R., et al (2001) Serotonergic dysfunction, negative mood states, and response to alcohol. Alcoholism, Clinical and Experimental Research, 25, 487-495.

Himle, J. A., Abelson, J. L., Haghightgou, H., et al (1999) Effect of alcohol on social phobic anxiety. American Journal of Psychiatry, 156, 1237-1243.

Keaney, K., Strang, J., Gossop, M., et al (2001) A doubleblind randomized placebo-controlled trial of lofexidine in alcohol withdrawal: lofexidine is not a useful adjunct to chlordiazepoxide. Alcohol and Alcoholism, 36, 426-430.

Kessler, R. C., McGonagle, K. A., Zhao, S., et al (1994) Lifetime and 12-month prevalence of DSM-III-R psychiatric disorders in the United States. Archives of General Psychiatry, 51, 8-19.

- Crum, R. M., Warner, L. A., et al (1997) Lifetime cooccurrence of DSM-III-R alcohol abuse and dependence with other psychiatric disorders in the National Comorbidity Survey. Archives of General Psychiatry, 54, 313-321.

Kranzler, H. R., Burleson, J. A., Del Boca, F. K., et al (1994) Buspirone treatment of anxious alcoholics. Archives of General Psychiatry, 51, 720-734.

_- _- Brown, J., et al (1996) Fluoxetine treatment seems to reduce the beneficial effects of cognitive-behavioural therapy in type B alcoholics. Alcoholism, Clinical and Experimental Research, 20, 1534-1541.

Krausz, M., Degkwitz, P., Kühne, A., et al (1998) Comorbidity of opiate dependence and mental disorders. Addictive Behaviors, 23, 767-783.

Kushner, M. G., Sher, K. J. \& Beitman, B.D. (1990) The relation between alcohol problems and anxiety disorders. American Journal of Psychiatry, 147, 685-695.

Lesch, O. \& Walter, H. (1996) Subtypes of alcoholism and their role in therapy. Alcohol and Alcoholism, 31 (suppl. 1), 63-67.

Lingford-Hughes, A. R., Acton, P. D., Gacinovic, S., et al (1998) Reduced levels of the GABA-benzodiazepine receptor in alcohol dependency in the absence of grey matter atrophy. British Journal of Psychiatry, 173, 116-122.

Malizia, A. L., Cunningham, V. J, Bell, C. J., et al (1998) Decreased brain GABA A-benzodiazepine receptor binding in panic disorder. Archives of General Psychiatry, 55, 715720.

Merikangas, K. R., Mehta, R. L., Molnar, B. E., et al (1998) Comorbidity of substance use disorders with mood and anxiety disorders: results of the international consortium in psychiatric epidemiology. Addictive Behaviors, 23, 893907.

Nutt, D. J. (1999) Alcohol and the brain. Pharmacological insights for psychiatrists. British Journal of Psychiatry, 175, 114-119.

— \& Cowen, P. J. (1987) Diazepam alters brain 5HT function in man: implications for the acute and chronic effects of benzodiazepines. Psychological Medicine, 17, 601-607.

Pitman, R. K., Orr, S. P., Altman, B., et al (1996) Emotional processing during eye movement desensitization and reprocessing [EMDR] therapy of Vietnam veterans with post-traumatic stress disorder. Comprehensive Psychiatry, 37, 419-429.

Potokar, J., Coupland, N., Wilson, S., et al (1999) Assessment of GABA A benzodiazepine receptor (GBzR) sensitivity in patients on benzodiazepines. Psychopharmacology, 146, $180-184$

Quitkin, F. M., Rifkin, A., Kaplan, J., et al (1972) Phobic anxiety syndrome complicated by drug dependence and addiction. A treatable form of drug abuse. Archives of General Psychiatry, 27,159-162.

Raistrick, D. (2000) Management of alcohol detoxification. Advances in Psychiatric Treatment, 6, 348-355. 
Randall, C. L., Thomas, S. E. \& Thevos, A. K. (2000) Gender comparison in alcoholics with concurrent social phobia: implications for alcoholism treatment. American Journal of Addiction, 9, 202-215.

— - \& - (2001) Concurrent alcoholism and social anxiety disorder: a first step toward developing effective treatments. Alcoholism, Clinical and Experimental Research, $25,210-220$.

Regier, D. A., Farmer, M. E., Rae, D. S., et al (1990) Comorbidity of mental disorders with alcohol and other drug abuse. JAMA, 264, 2511-2518.

Rothbaum, B. O. (1997) A controlled study of eye movement desensitization and reprocessing in the treatment of posttraumatic stress disordered sexual assault victims. Bulletin of the Menninger Clinic, 61(3), 317-334.

Roy-Byrne, P. P., Lewis, N., Villacres, E., et al (1989) Preliminary evidence of benzodiazepine subsensitivity in panic disorder. Biological Psychiatry, 26, 744-748.

Rudolph, U., Crestani, F. \& Mohler, H. (2001) GABAA receptor subtypes: dissecting out their pharmacologica functions. Trends in Pharmacological Sciences, 22, 188-194.

Saunders, J. B., Aasland, O. G., Babor, T. F., et al (1993) Development of the Alcohol Use Disorders Identification Test (AUDIT): WHO Collaborative Project on Early Detection of Persons with Harmful Alcohol Consumption - II. Addiction, 88, 791-804.

Schneier, F. R., Goetz, D., Campeas, R., et al (1998) Placebocontrolled trial of moclobemide in social phobia. British Journal of Psychiatry, 172, 70-77.

Scott, J., Gilvarry, E. \& Farrell, M. (1998) Managing anxiety and depression in alcohol and drug dependence. Addictive Behaviors, 23, 919-931.

Versiani, M. Nardi, A. E., Mundim, F. D., et al (1992) Pharmacotherapy of social phobia. A controlled study with moclobemide and phenelzine. British Journal of Psychiatry, 161, 353-360.

World Health Organization (1992) The ICD-10 Classification of Mental and Behavioural Disorders. Geneva: WHO.

\section{Multiple choice questions}

1. As regards anxiety disorders:

a social anxiety disorder commonly occurs as a consequence of alcoholism

b tricyclic antidepressants are particularly efficacious in treating social anxiety disorder c chronic use of alcohol reduces anxiety

$\mathrm{d}$ of all anxiety disorders, social anxiety is the least associated with alcoholism

e about $30 \%$ of patients with social anxiety misuse alcohol.

2. As regards substance misuse:

a in opiate addicts, anxiety is seen more frequently than depression

b the self-medication hypothesis explains only some comorbid substance use

c misuse of stimulants can result in generalised anxiety disorder

d intoxication with hallucinogens commonly results in anxiety

e withdrawal from cannabis is associated with anxiety.

3. Studies suggest that:

a reduced noradrenergic function underlies some features of withdrawal from alcohol and opiates

b Type 1 alcoholism is associated with low levels of 5-HT

c hypofunction of the GABA-ergic system is proposed to underlie both alcoholism and some anxiety disorders

$\mathrm{d}$ alcohol is a GABA-benzodiazepine receptor agonist

e mCPP induces craving in Type 2 alcoholism.

4. In treating anxiety disorders:

a venlafaxine is the drug treatment of choice for panic disorder

b citalopram is licensed for social anxiety disorder

c moclobemide is licensed for social anxiety disorder

d SSRIs have been shown to be efficacious in treating anxiety complicated by alcoholism

e treating the anxiety and substance misuse simultaneously has been shown to be the best strategy.

5. Evidence suggests that:

a patients with panic disorder are supersensitive to the effects of benzodiazepines

b lofexidine ameliorates some symptoms of alcohol withdrawal

c anxiety is a predictor of response to acamprosate

d CBT seems to be more effective than 12-step therapy in treating male alcoholism complicated by anxiety

e abstinence from alcohol alone rarely improves anxiety.

\section{MCQ answers}

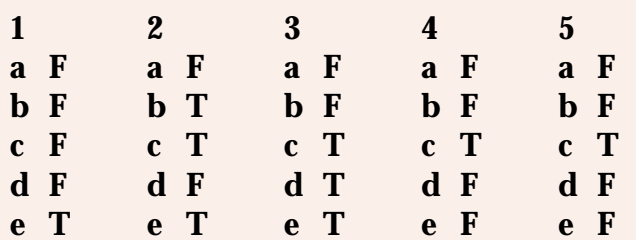

